Simulation of Main-Chamber Recycling in DIII-D with the

UEDGE Code

M.E. Rensink, M. Groth, G.D. Porter, T.D.

Rognlien, J.G. Watkins

April 26, 2006

Plasma Surface Interactions 17

Hefei, China

May 22, 2006 through May 26, 2006 
This document was prepared as an account of work sponsored by an agency of the United States Government. Neither the United States Government nor the University of California nor any of their employees, makes any warranty, express or implied, or assumes any legal liability or responsibility for the accuracy, completeness, or usefulness of any information, apparatus, product, or process disclosed, or represents that its use would not infringe privately owned rights. Reference herein to any specific commercial product, process, or service by trade name, trademark, manufacturer, or otherwise, does not necessarily constitute or imply its endorsement, recommendation, or favoring by the United States Government or the University of California. The views and opinions of authors expressed herein do not necessarily state or reflect those of the United States Government or the University of California, and shall not be used for advertising or product endorsement purposes. 


\title{
Simulation of main-chamber recycling in DIII-D with the UEDGE code
}

\author{
M. E. Rensink ${ }^{\mathrm{a},{ }^{*}}$, M. Groth ${ }^{\mathrm{a}, \mathrm{b}}$, G. D. Porter ${ }^{\mathrm{a}}$, T. D. Rognlien ${ }^{\mathrm{a}}$ and J. G. Watkins ${ }^{\mathrm{b}, \mathrm{c}}$ \\ ${ }^{a}$ Lawrence Livermore National Laboratory, Livermore, California, USA \\ ${ }^{\mathrm{b}}$ General Atomics, La Jolla, California, USA \\ 'Sandia National Laboratory, Albuquerque, New Mexico, USA
}

This report demonstrates a computer simulation model for single-null diverted plasma configurations that include simultaneous interaction of the scrape-off layer (SOL) plasma with toroidally symmetric main-chamber limiter surfaces and divertor plate surfaces. The simulations use the UEDGE code which treats the SOL plasma and recycled neutrals as twodimensional toroidally symmetric fluids. The spatial domain can include field lines that intersect main chamber surfaces in the far scrape-off layer, which allows the model to include simultaneous plasma contact with both divertor and main chamber targets. Steady-state simulation results for low-density L-mode plasma discharges in DIII-D show that total core fueling increases by about 70 percent when the separatrix-baffle gap is reduced from $6 \mathrm{~cm}$ to $3 \mathrm{~cm}$. The additional core fueling is due to neutrals which originate from the ion particle flux incident on the upper outer divertor baffle.

JNM keywords:

PACS:

PSI-17 keywords:

"Corresponding Author Address: mail stop L-637, P.O. Box 808, Livermore, CA 94551, USA

"Corresponding Author e-mail: rensink1@ @llnl.gov

Presenting author: Mathias Groth

Presenting author e-mail: groth@ fusion.gat.com 


\section{INTRODUCTION}

The poloidal divertor is currently the preferred configuration for power and particle exhaust from a tokamak plasma. Ideally, all plasma contact with material surfaces occurs in a remote divertor chamber which accomodates high heat flux and particle flux. However, if the radial gap between the separatrix and the main chamber wall is not large enough, the SOL plasma can interact with nearby "limiter" surfaces to produce high recycling of neutral hydrogen near the confined plasma and uncontrolled fueling of the core. Observations on several tokamaks suggest that this main-chamber recycling (MCR) can be significant [1].

The primary purpose of this paper is to demonstrate a computational model for simulating main chamber recycling. This model was previously used [2] to simulate plasma startup in ITER from an initial limiter configuration to a final single-null divertor configuration. An alternative model for MCR simulation has been described in Reference [3]. A secondary purpose of this report is to compare simulation results for various gap sizes and plasma densities as a guide to interpreting experimental observations on core fueling, D-alpha radiation, SOL plasma profiles and global particle balance. Detailed comparisons of our results with experimental data are not yet appropriate because some potentially significant effects have been omitted from the simulations, namely, cross-field drifts, impurities and divertor geometry. These omissions allow the code to run more robustly on a mesh with sufficient spatial resolution to accurately model simultaneous recycling at both the upper baffle and divertor plates in DIII-D lower-single-null configurations.

\section{MODEL}

The two-dimensional fluid code UEDGE [4] is used to calculate toroidally symmetric 
solutions for the SOL plasma in lower-single-null configurations of DIII-D. Transport along field lines is assumed to be purely classical with flux limits imposed to account for kinetic effects; cross-field drifts are not included in these simulations, although the code does have this capability. Generally, the drifts introduce more non-linearity in the plasma response and relaxation to a steady state, so the solution algorithm becomes less robust. We include anomalous radial transport; the simulations in this report use a purely diffusive model with particle diffusivity $=0.65 \mathrm{~m}^{\wedge} 2 / \mathrm{sec}$ and thermal diffusivity $=2.60 \mathrm{~m}^{\wedge} 2 / \mathrm{sec}$; these values produce the best agreement between UEDGE simulations and a large set of expermental data [5]. The simulations in this report do not include an anomalous convective radial velocity. Neutral particles from recycling are also represented by a fluid model; a parallel momentum balance equation for neutrals is included. Neutrals may also be modeled with the DEGAS2 Monte Carlo code [6] using the background plasma from UEDGE.

The spatial mesh for the simulation is based on poloidal magnetic flux surfaces generated by the MHD equilibrium code EFIT. A second set of surfaces, roughly orthogonal to the magnetic flux surfaces, completes the mesh. The mesh may include field lines that terminate on the upper baffle which allows simultaneous plasma contact with both lower divertor targets and the main chamber wall (upper baffle) as shown in Figure 1. In this report we simulate two DIII-D plasma configurations, shown in Figure 2, with $3 \mathrm{~cm}$ and $6 \mathrm{~cm}$ gaps. The gap is defined as the radial distance between the separatrix flux surface and the flux surface which just touches the upper outer baffle, measured at the outboard midplane. The outermost flux surface in all simulations is at a normalized poloidal magnetic flux of psi0max $=1.13$ which corresponds to a radial distance of about $6 \mathrm{~cm}$ at the outboard midplane. In the large gap case, this flux surface just misses the upper baffle/limiter; in the small gap case, all flux surfaces beyond psi0=1.07 intersect the limiter. For the small-gap simulations we have used a modified wedge-shaped limiter to represent the upper outer baffle in DIII-D, as shown in 
Figure 2a.

\section{SIMULATION RESULTS}

We examine the effect of gap size and core density on core fueling. In our simulations, low density means the core plasma density is $1 \mathrm{e} 19 / \mathrm{m}^{\wedge} 3$ on innermost magnetic flux surface of the mesh; this density corresponds to about 25 percent of the Greenwald [7] density in the experiment. Our high density case corresponds to about 50 percent of the Greenwald density.

We quantify the core fueling as the total neutral particle flux (in Amps) across the separatrix into the core region of our simulations. The effect of a smaller gap is to increase the core fueling because there is a recycling source at the limiter close to the separatrix. For our simulations we find that the core fueling increases by 70 percent (from 180 Amps to 310 Amps) as the gap size is reduced from $6 \mathrm{~cm}$ to $3 \mathrm{~cm}$. This is shown in Figure 3 which gives the neutral particle flux across the separatrix for small and large gap size. The strong peak near the upper baffle/limiter in the small gap case is responsible for the increased in core fueling. In the experiment, increased core fueling should manifest itself by a reduction in the gas puff needed to maintain a fixed plasma density. Since it is not known how much of the gas puff contributes directly to core fueling, one can not quantify the gas puff reduction in terms of the increased core fueling in this simulation. The increased core fueling in the small gap case is evident in Figure 4 which shows the local D-alpha emissivity in the vicinity of the upper baffle. Peak values of the emissivity $\left(1 \mathrm{e} 20\right.$ photons $\left./ \mathrm{sec} / \mathrm{m}^{\wedge} 3\right)$ near the limiter surface are about two orders of magnitude smaller than peak values in the divertor regions, but should be observable in experiment. Measurements with toroidally-viewing TV cameras in DIII-D are sometimes masked by reflected light, so a direct comparison between simulation and experiment is not possible at this time. The gap effect persists at higher density, but it is weaker because the SOL plasma more effectively screens the core plasma from neutrals; We 
find that at high density the core fueling only increases by 20 percent (from 90 Amps to 110 Amps) as the gap size is reduced.

Both midplane and divertor plasma profiles are affected by the gap size, especially on shadowed flux surfaces, as shown in Figure 5. For the small gap case the density in the outboard midplane is lower than in the large gap case. Divertor recycling is also reduced when the gap size is reduced because the sonic plasma flow toward the baffle/limiter competes with the flow toward divertor plates on shadowed flux surfaces. The baffle acts as a nearby target plate for some particles which would have flowed along the outermost flux surfaces towards the divertor. This can be seen in the ion saturation current profiles of Figure $5 \mathrm{~b}$ where the total current to the outer divertor target plate decreases from $7.9 \mathrm{kA}$ to $6.6 \mathrm{kA}$ when the gap size is reduced. The effect is even stronger at higher density with the current decreasing from $16.2 \mathrm{kA}$ to $9.2 \mathrm{kA}$. Similar changes occur at the inner divertor target plates.

The modified plasma profiles in the far SOL are a result of plasma flow toward the baffle/limiter, as shown in Figure 6. For the large gap case the poloidal flow along the outermost flux surfaces is nearly stagnant in the vicinity of the upper baffle position; most of the plasma flows toward the divertor plates. For the small gap case, a Bohm sheath condition applies at the limiter surface, so the plasma flow on shadowed flux surfaces is directed from each midplane toward the limiter. This sonic flow to the upper baffle target competes with the flow to the divertor targets, so the recycling flux at the divertor plates decreases with the gap size. The size of the effect is small (about 15-20 percent), but is consistent with D-alpha and probe measurements in the DIII-D divertor.

The ion current to the outer wall also decreases for reduced gap size because some ions are lost by flow along field lines to the baffle/limiter before they have a chance to diffuse radially 
across the flux surfaces to the vessel wall. At low density there is a 55 percent reduction in the current to the wall, while at higher density the reduction is 40 percent.

For the low density, small gap case we also used the DEGAS2 code to simulate neutrals that originate from recycling sources at the divertor plates and at the upper baffle. This tests the validity of the fluid neutral model in UEDGE. The (fixed) background plasma for this test is given by the UEDGE simulation result. The halo region between the UEDGE mesh and the DIII-D vacuum vessel was not included in the simulation. The total core fueling from the DEGAS2 simulation was 204 Amps compared to 310 Amps from the UEDGE fluid neutral simulation; this discrepancy may be due to kinetic effects when the mean-free-path of the neutrals becomes large compared to the plasma scale length, and to the UEDGE assumption that the neutrals and ions share a common temperature at each point in the simulation domain. In the UEDGE simulation there is also a small contribution from ion recycling at the vessel walls which the DEGAS2 simulations do not include. The MCR contribution to total core fueling is 34 percent according to DEGAS2 and 40 percent according to UEDGE.

\section{SUMMARY}

We have obtained steady-state plasma solutions for a magnetic configuration in which recycling occurs simultaneously at divertor plates and on a main chamber limiter surface. The most significant effect of reduced gap size is increased core fueling by neutrals which originate from recycling on the limiter. This is particularly important at low density where the SOL plasma is relatively transparent to the neutrals. The increased Dalpha emissivity of the plasma in the vicinity of the upper baffle should be observable. The simulations show that the gap size has a weak effect on the radial profiles of the SOL plasma at the outboard midplane and divertor plates. There is no sign of a sharp "break" in the profiles at the shadow 
boundary. This agrees qualitatively with Thomson scattering and reciprocating probe data for these cases. [A Langmuir probe at the surface of the baffle indicates ....?] Note that in all cases the particle and power flow to the limiter is small compared to that on the divertor plates.

\section{ACKNOWLEDGEMENTS}

This work was performed under the auspices of the US Department of Energy under contract nos. W-74050-ENG-48 (LLNL), DE-AC03-99ER54463 (DIII-D) and DE-AC04-94AL85000 (SNL).

\section{REFERENCES:}

[1] D.G. Whyte, B.L. Lipschultz, P.C. Stangeby, J. Boedo et al., Plasma Phys. Control. Fusion 47 (2005) 1579-1607.

[2] M.E. Rensink and T.D. Rognlien, J. Nucl. Mater. 266-269 (1999) 1180.

[3] S. Lisgo, P.C. Stangeby, A.G. McLean, J.D. Elder et al., Bull. Amer. Phys. Soc. xx (2005) xxx.

[4] T.D. Rognlien et al., Contrib. Plasma Phys. 34 (1994) 362.

[5] M. Groth, G.D. Porter, N.H. Brooks, M.E. Fenstermacher et al., "Comprehensive comparison of measurements and modeling of the SOL in low density L-mode plasmas in DIII-D", submitted to Nuclear Fusion (2005). 
[6] D.P. Stotler et al., Contrib. Plasma Phys. 34 (1994) 392.

[7] M. Greenwald, et al., Nucl. Fusion 28 (1988) 2199.

\section{FIGURES:}

1. Global view of the simulation region relative to the DIII-D vacuum vessel. A simplified wedge-shaped limiter replaces the upper divertor baffle. There is a halo region between the outermost flux surface and the vacuum vessel that is not not included in these simulations. Simplified orthogonal divertor plates are used in this report.

2. Details of the mesh near the upper baffle/limiter for (a) small gap and (b) large gap simulations. The mesh is non-uniform in both radial and poloidal directions; in the small gap case this helps resolve steep plasma gradients near the limiter tip.

3. Neutral particle flux (core fueling) current profile at the separatrix. The horizontal axis gives the distance along the separatrix starting at the x-point and going in a clockwise direction around the core plasma. For the small gap case a strong peak is evident near the upper baffle/limiter.

4. Color contours of the D-alpha emissivity in a poloidal plane for the small gap case. The emissivity near the limiter tip is about two orders of magnitude smaller than the peak emissivity near the divertor plates. 
5a. Plasma density profiles in the outboard midplane for small and large gap sizes. The vertical dashed line shows the position of the flux surface that just touches the limiter tip in the small gap case; it separates the SOL from the shadowed region.

5b. Ion saturation current profiles at the outboard divertor plate; the horizontal axis denotes the position of the flux surface at the outboard midplane; The vertical dashed line shows the position of the flux surface that just touches the limiter tip in the small gap case; it separates the SOL from the shadowed region.

6. The poloidal flow velocity of the plasma versus distance along a flux surface in the far SOL. For the large gap case, the flow is nearly stagnant at the upper baffle/limiter position; in the small gap case the flow changes sign across the limiter because Bohm sheath conditions are applied at the limiter surface. 
Figure 1

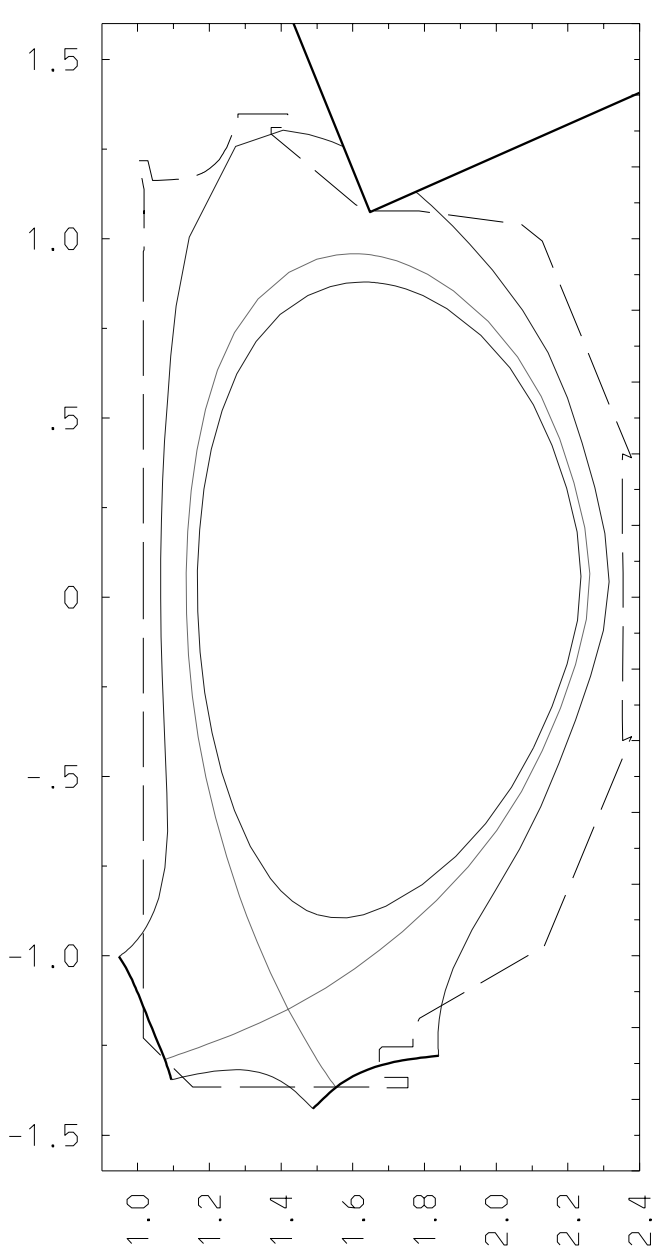


Figure 2
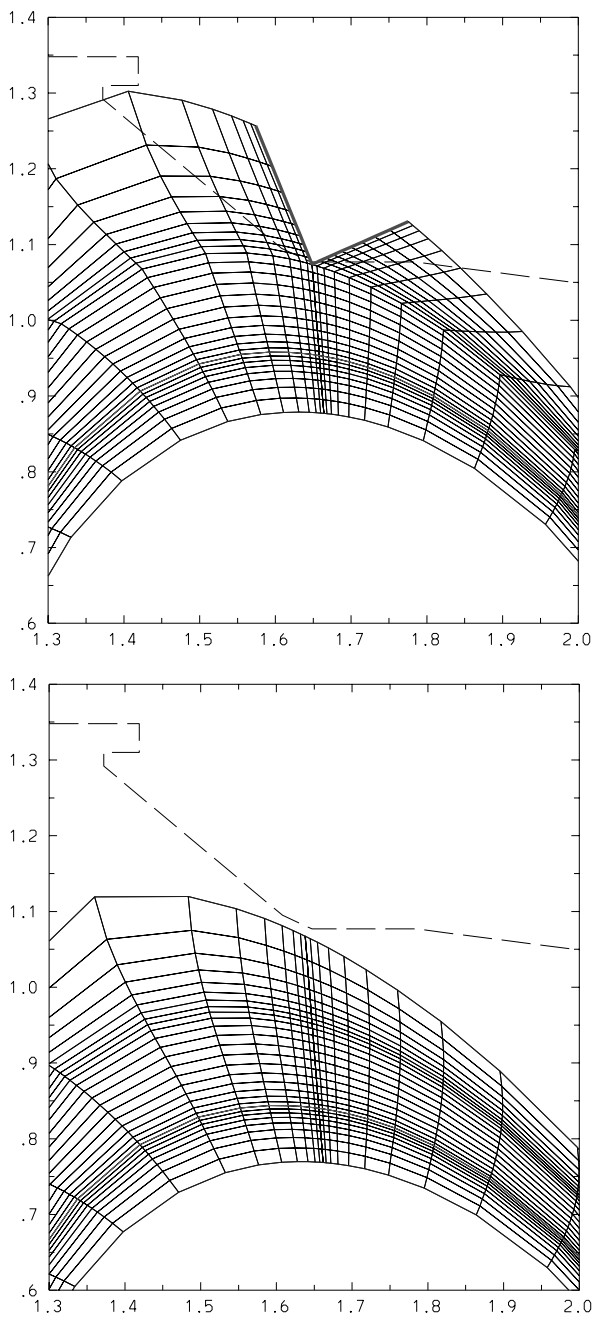


\section{Figure 3}

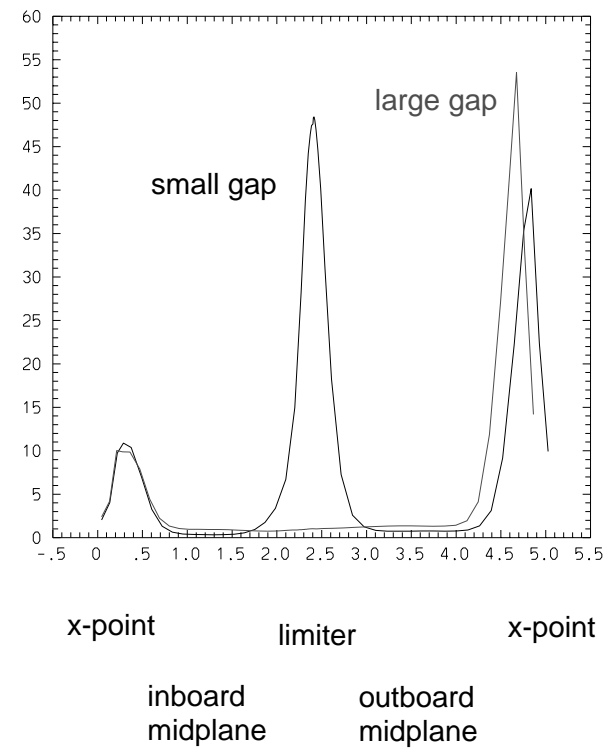


Figure 4

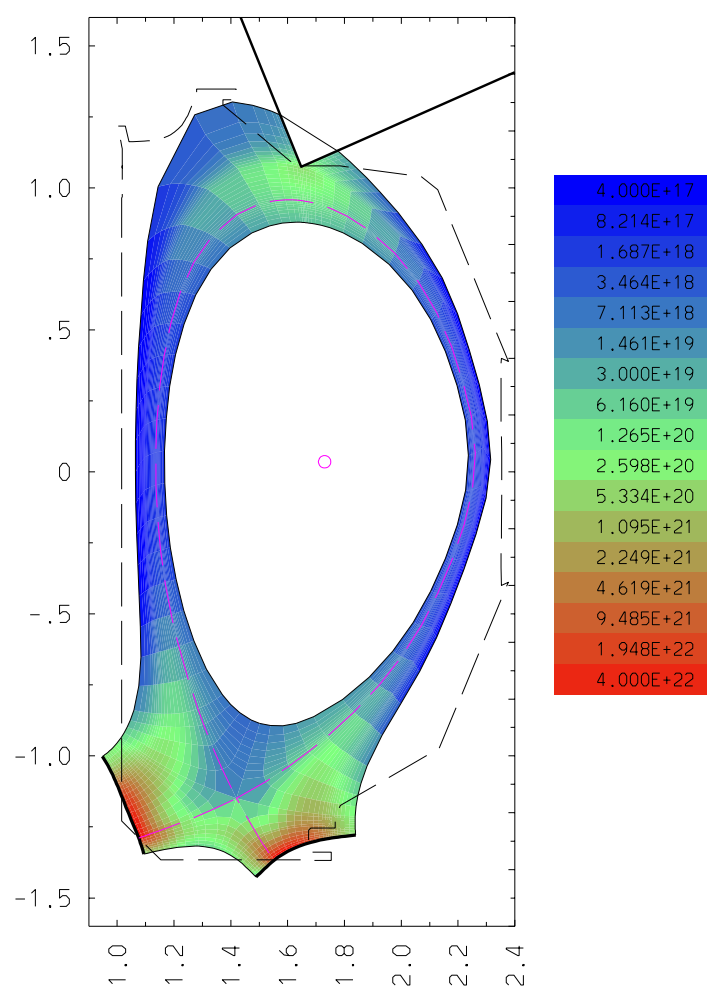


Figure 5
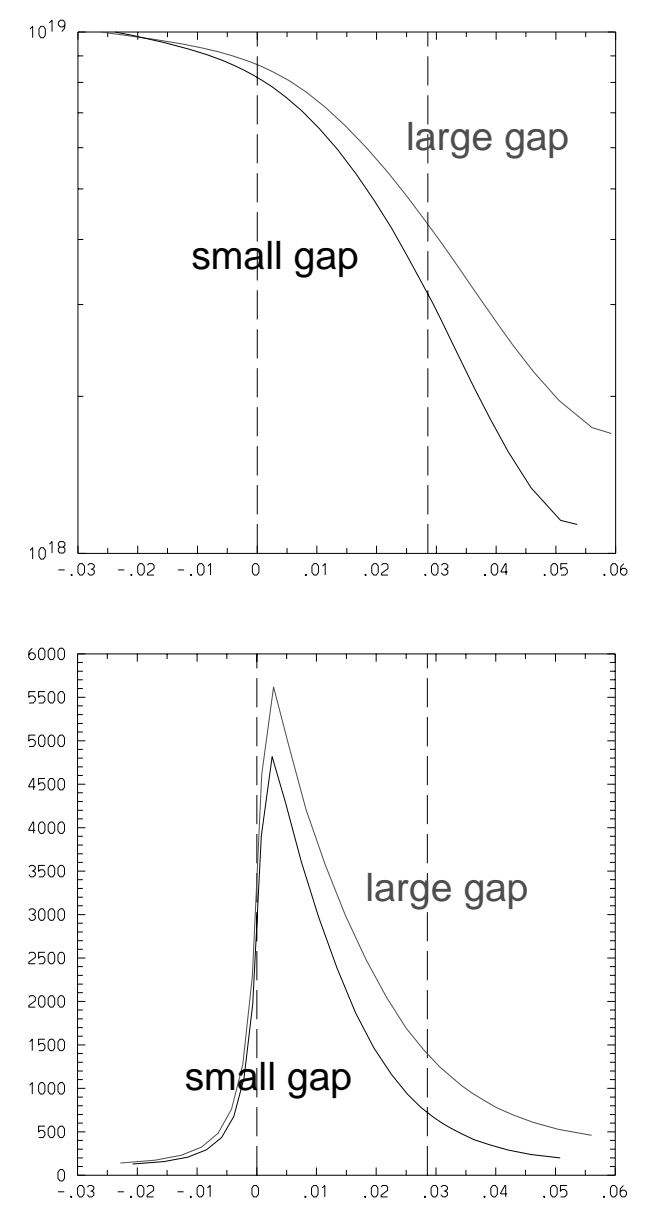
Figure 6

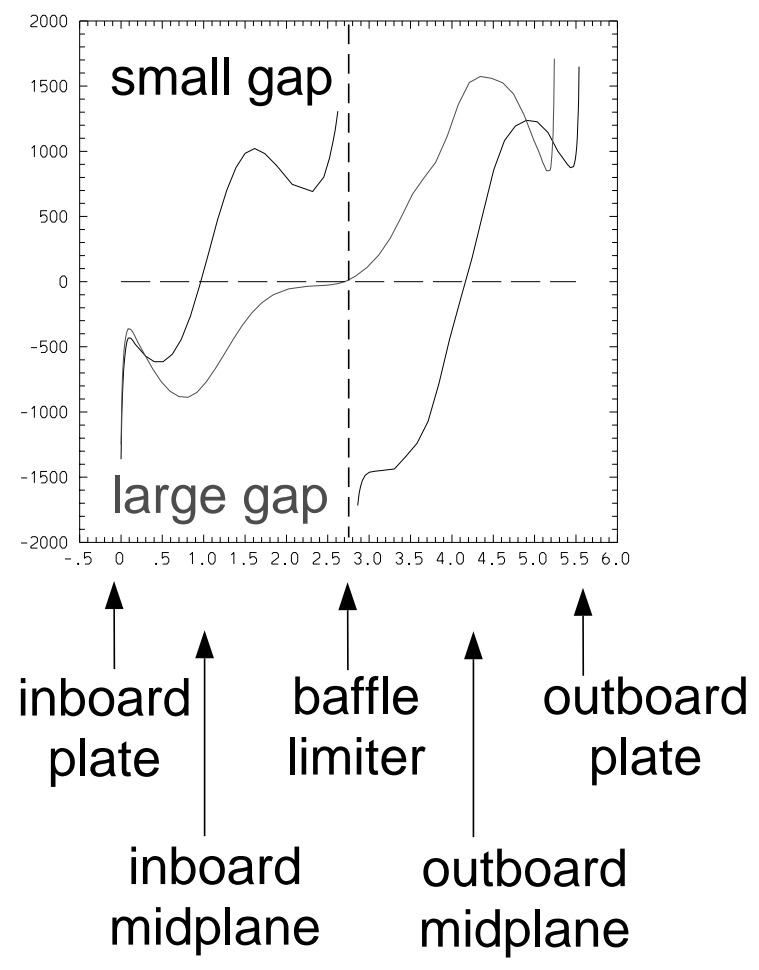

